\title{
Shellability of Pointed Integer Partition
}

\author{
Samuel Asefa Fufa ${ }^{1 *}$, Ayichew Abebe Gebeyehu ${ }^{1}$ \\ 1 Department of Mathematics, Adiss Ababa University, Ethiopia Bonga Collages of Teachers Education
}

\begin{abstract}
In this paper we study, pointed integer partition defined as a pair $\{u, \underline{m}\}=\left\{u_{1}, u_{2}, \cdots, u_{r}, \underline{m}\right\}$ where $u=\left\{u_{1}, u_{2}, \cdots, u_{r}\right\}$ is an integer partition of $n-m$, and $m$ is a non-negative integer $\leq n$. Shellability of pointed integer partition with Möbius values -1 and +1 denoted by $R_{n}$. We determine the cardinality of $I_{n}^{*}$ and $R_{n}$ for $1 \leq n \leq 10$ and $n \geq 1$ respectively and compute the Möbius number of $I_{n}^{*}$ for $1 \leq n \leq 6$. We have shown hat $R_{n}$ admit an EL-labeling which is EL-shellable.
\end{abstract}

Keywords: Pointed Integer Partition, Hassee Diagram, Möbius Values and EL-Shellability.

\subsection{Notations}

\section{Preliminary}

Through out this paper we will use the following notations:

1) $[n]=\{1,2,3, \cdots, n\}$.

2) $|X|=$ Cardinality of $X$.

3) Partially ordered set (or poset) $\equiv$.

4) $\preceq$ indicates cover relation.

5) $\bigcirc$ indicates vertex of the Hasse diagram.

6) On the Hasse diagram marked by red color $0,+1$ or -1 are Möbius numbers.

7) $\hat{0}$ and $\hat{1}$ stand minimal and maximal elements of poset respectively.

8) $\mu$ denoted Möbius function.

\subsection{Partially Ordered Sets and Möbius Function}

\subsubsection{Equivalence Relations and Partitions}

Definition 1.2.1. A binary relation $R$ on a set $X$ is said to be

a. reflexive if $x R x$ for all $x$ in $X$,

b. symmetric if $x$ Ry implies $y R x \forall x, y \in X$,

c. transitive if $x R y$ and $y R z$ imply $x R z \forall x, y, z \in X$.

$A$ relation $R$ is called an equivalence relation if it is reflexive, symmetric and transi-tive, and in this case, we say that $x$ and $y$ are equivalent, if $x R y$.

Definition 1.2.2. For an equivalence relation $R$ on a set $A$, the set of the elements of $A$ that are related to an element, say $a$, of $A$ is called the equivalence class of element $a$ and it is denoted by [a].

Definition 1.2.3. A partition of a positive integer $n$ is a way of writing $n$ as a sum of positive integers. The summands of the partition are known as parts.

\subsubsection{Partially Ordered Sets}

Definition 1.2.4. A partially ordered set $P$ (or poset, for short) is a set (which by abuse of notation we also call $P$ ), together with a binary relation denoted $\leq$ (or $\leq_{p} p$ when there is a possibility of confusion), satisfying the following three axioms:

1. For all $t \in P, t \leq t$ (reflexivity)

2.If $s \leq t$ and $t \leq s$, then $s=t$ (antisymmetry)

3. If $s \leq t$ and $t \leq u$, then $s \leq u$ (transitivity)

We use the obvious notation $t \geq s$ to mean $s \leq t, s<t$ to mean $s \leq t$ and $s \neq t$, and $t>s$ to mean $s<t$. We say that two elements $s$ and $t$ of $P$ are comparable if $s \leq t$ or $t$ 
$\leq s$; otherwise $s$ and $t$ are incomparable, denoted $s \| t$ [10].

Similarly the open interval $(x, y)$ can be defined as $(x, y)=\{z \in P: x<z<y\}$ : If $x, y \in P$, then we say $y$ covers $x$ if $x<y$ and if no $z \in P$ satisfies $x<z<y$. Thus $y$ covers $x$ if and only if $x<y$ and $[x, y]=\{x, y\}$. Finite posets are represented graphically by the Hasse diagram, which is drawn using elements of $P$ as vertices and the cover relation as edge (directed from below). For instance the Hasse diagram of poset $B_{3}$ consisting of the subsets of the set $[3]=\{1,2,3\}$ ordered by inclusion.

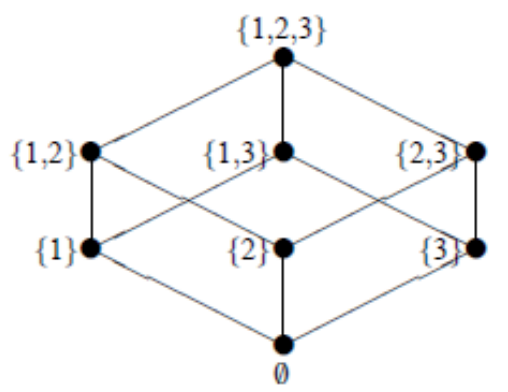

Fig. 1: The Hasse Diagram of [3].

We say that a poset $P$ has a minimal element denoted by $\hat{0}$ if there exists an element $\hat{0} \in P$ such that $x \geq \hat{0}$ for all $x$ in $P$. Similarly, $P$ has a maximal element denoted by $\hat{i}$ if there exists $\hat{1}$ in $P$ such that $x \hat{1}$ for all $x$ in $P$ [10].

Definition 1.2.5.

1. An element $a$ in a poset $(S, \leq)$ is called maximal if it is not less than any other element in $S$. That is: $\nexists b \in S(a \leq b)$, if there is one unique maximal element $a$, we call it the maximum element (or the greatest element).

2. An element $a$ in a poset $(S, \leq)$ is called minimal if it is not greater than any other element in $S$. That is: $\nexists b \in S(a \leq b)$, if there is one unique minimal element $a$, we call it the minimum element (or the least element).

Definition 1.2.6. A lattice is a partially ordered set in which every pair of elements has both a least upper bound and a greatest lower bound.

\section{Definition 1.2.7.}

1. A chain of a partially ordered set $P$ is a totally ordered subset $C \subseteq P$ i.e. $C=\left\{x_{0}, \cdots, x_{l}\right\}$ with $\left\{x_{0} \preceq x_{1} \preceq \cdots \leq x_{l}\right\}$.

The quantity $\ell=|C|-1$ is its length and is equal to the number of edges in its Hasse diagram.

2. A chain is maximal if no other chain strictly contains it.

3. The rank of $P$ is the length of the longest chain in $P$. 4. $P$ is graded if all maximal chains have the same length.

\subsubsection{Hasse Diagram}

Definition 1.2.8. The Hasse diagram of a partially ordered set $P$ is the (directed) graph whose vertices are the elements of $P$ and whose edges are the pairs $(x, y)$ for which $y$ covers $x$. It is usually drawn so that elements are placed higher than the elements they cover [6].

With the cover relation at hand, we can get a diagrammatic representation of the partially ordered set (poset). Let us consider $x$ and $y$, and assume that $x \preceq y$. Then we draw $x$ in a vertical plane below $y$ and connect both with a straight line. This is repeated for every ordered pair, i.e., for all pairs of two objects for which $\preceq$ relation holds. The resulting diagram is denoted as Hasse diagram (sometimes partial order set diagram, order diagram, line diagram, or simply the diagram) after the German Mathematician Hasse, who made this kind of visualization popular.

\subsubsection{The Möbius Function of a Partially Ordered Set}

Definition 1.2.9. Let $P$ be a finite partially ordered set, and let $f$ and $g$ be functions on $P$, thus $f$ and $g$ are related by the formula [10].

$$
f(x)=\sum_{y \leq x} g(y), \quad \forall x \text { in } P .
$$

By elementary reasoning, one concludes that the values of $g$ can be expressed as integral linear combinations of the values of $f$, i.e. $g(x)=f(x)$ if $x$ is a minimal element, $g(x)=f(x)-f(y)-f(z)-f(w)-2 f(u)$ if the ideal generated by $x$ is as shown in figure below.

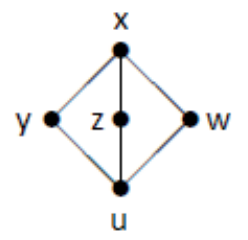

Indeed, for each $x$ in $P$ there is a formula

$$
g(x)=\sum_{y \leq x} \mu_{p}(y, x) f(y)
$$

where $\mu_{p}(y, x)$ is a unique integer valued function on $P \times P$, depending only on $P$ (not on $f$ or $g$ ), assuming nonzero values only when $y \leq x$. The function $\mu_{p}$ is called the Möbius function of $P$, and 
(2) is known as the Möbius inversion formula.

Definition 1.2.10. The Möbius function $\mu$ is a function that assigns to each interval in a poset $p$ and its recursive formulation is given by

$$
\mu(x, y)= \begin{cases}1 & \forall x=y \\ -\sum_{z: x \leq z<y} \mu(x, y), & \forall x<y .\end{cases}
$$

Example 1.2.1. Find Möbius function of Figure 1

$$
\begin{aligned}
& \mu(\emptyset)=\mu(\hat{0})=1, \\
& \mu(\{1\})=\mu(\{2\})=\mu(\{3\})=-\mu(\emptyset)=-1, \\
& \mu(\{1,2\})=-[\mu(\emptyset)+\mu(\{1\})+\mu(\{2\})]=-(1-1-1)=1, \\
& \mu(\{1,3\})=-[\mu(\emptyset)+\mu(\{1\})+\mu(\{3\})]=-(1-1-1)=1, \\
& \mu(\{2,3\})=-[\mu(\emptyset)+\mu(\{2\})+\mu(\{3\})]=-(1-1-1)=1, \\
& \mu(\{1,2,3\})=-\left[\begin{array}{l}
\mu(\emptyset)+\mu(\{1\})+\mu(\{2\})+\mu(\{3\})+]= \\
+\mu(\{1,2\})+\mu(\{1,3\})+\mu(\{2,3\})
\end{array}\right]= \\
& =-(1-1-1-1+1+1+1)=-1
\end{aligned}
$$

\subsection{Set Partition}

Definition 1.3.1. A set partition $\pi$ of a set $S$ is a collection $B_{1}, B_{2}, \cdots B_{k}$ of nonempty disjoint subsets of $S$ such that $\bigcup_{i=1}^{k} B_{i}=S$. The elements of a set partition are called blocks, and the size of a block $B$ is given by $|B|$ the number of elements in $B$ [5].

Definition 1.3.2. Let $\pi$ be any set partition of the set $[n]=\{1,2, \cdots, n\}$. We represent $\pi$ in either sequential or canonical form. In the sequential form, each block is represented as sequence of increasing numbers and different blocks are separated by the symbol $/$. In the canonical representation, we indicate for each integer the block in which it occurs, that is, $\pi=\pi_{1} \pi_{2} \cdots \pi_{n}$ such that $j \in B_{\pi j}, 1 \leq j \leq n$ [5]

We denote the set of all set partitions of $[n]$ by $P_{n}=P([n])$, and the number of all set partitions of $[n]$ by $P_{n}=\left|P_{n}\right|$, with $P_{0}=1$ (as there is only one set partition of the empty set). Also, we denote the set of all set partitions of $[n]$ with exactly $k$ blocks by $P_{n, k}$.

Example 1.3.1. The set partitions of [3] in sequential form are 1/2/3,1/23,12/3,13/2, and 123, while the set partitions of [3] in canonical representation are 123, $122,112,121$, and 111, respectively. Thus, $P_{3}=5$.

Example 1.3.2. The set partition $14 / 257 / 3 / 6$ has canonical form 1231242. We have that $\pi_{1}=\pi_{4}=1$, as both 1 and 4 are in the first block. Likewise, $\pi_{2}=\pi_{5}=\pi_{7}=2$, as 2 , 5 , and 7 are in the second block.
Theorem 1.3.1. Let $P_{n}$ be the number of set partitions of $[n]$. Then Pn satisfies the recurrence relation [5].

$P_{n}=\sum_{j=0}^{n-1}\left(\begin{array}{c}n-1 \\ j\end{array}\right) P_{j} \quad$ with initial condition $\quad P_{0}=1$

Definition 1.3.3. The number of set partitions of $[n]$ into $k$ blocks is denoted by $S(n, k)$ or $\left\{\begin{array}{l}n \\ k\end{array}\right\}$. The values $S(n, k)$ are called Stirling numbers of the second kind.

Theorem 1.3.2. The number of set partitions of $[n]$ into $k$ blocks satisfies the recurrence [5]

$S(n+1, k)=k S(n, k)+S(n, k-1)$

with $S(1,1)=1, S(n, 0)=0$ for $n \geq 1$, and $S(n, k)=0$ for $n<k$.

\section{Pointed Integer Partition}

In this section, we have used [2], [7] and [8] to find the cardinality for $1 \leq n \leq 10$, Hasse diagram and Möbius function of pointed integer partition for $1 \leq n \leq 6$.

\subsection{Introduction to Pointed Integer partition}

Let $n$ be a non-negative integer. A multiset $u=\left\{u_{1}, u_{2}, \cdots u_{r}\right\}$ of integers is an integer partition of $n$ provided that either $n=0$ and $u=\{0\}$ or $n \geq 1$ and

(a) $\sum_{i=1}^{r} u_{i}=n$

(b) $u_{i} \geq 1$, for all $i=1,2, \cdots, r$.

Here we regard the set $u$ as a multiset of positive integer which are unordered. Hence a partition of $n$ is a representation of $n$ as a sum of integers where the order of the terms (or parts) is irrelevant. We use multiplicities as a superscript of each $u_{i}$ in their decreasing order to give the multiset $u$. Thus, for instance, for partition of $23,\{6,4,4,3,2,2,1,1\}=\left\{6,4^{2}\right.$, $\left.3,2^{2}, 1^{2}\right\}$ [8].

Definition 2.1.1. A pair $\{u, \underline{m}\}=\left\{u_{1}, u_{2}, \cdots, u_{r}, \underline{m}\right\}$ is called a pointed integer partition of $n$ if $u=\left\{u_{1}, u_{2}, \cdots, u_{r}\right\}$ is an integer partition of $n-m$, where $m$ is a non-negative integer $\leq n$. The integer $m$ is called the pointed part. It is underlined to distinguish it from the other parts of the partition, and we write $u_{1} u_{2} \cdots u_{r} \underline{m}$ to denote a pointed integer partition $\{u, \underline{m}\}[8]$.

Let $I_{n}^{\cdot}$ denote the set of all pointed integer partitions of the non-negative integer $n$.

Partially order the set $I_{n}^{*}$ by the two cover relations: 
(1)

$$
\begin{aligned}
& \left\{u_{1}, \cdots, u_{i}, \cdots, u_{j}, \cdots, u_{r}, \underline{m}\right\} \\
& \leq\left\{u_{1}, \cdots, \hat{u}_{i}, \cdots, \hat{u}_{j}, \cdots, u_{r}, u_{i}+u_{j}, \underline{m}\right\}
\end{aligned}
$$

and

(2)

$\left\{u_{1}, u_{2}, \cdots, u_{i}, \cdots, u_{r}, \underline{m}\right\}$

$$
\leq\left\{u_{1}, u_{2}, \cdots, \hat{u}_{i}, \cdots, u_{r}, \underline{u_{i}+m}\right\} \text {. }
$$

Here $\hat{u}_{1}$ and $\hat{u}_{j}$ means that the corresponding elements are omitted.

In [8], the poset of pointed integer partition $I_{n}^{*}$ of $n$ was introduced and the study of its cardinality was not delt, the Möbius function and the Hasse diagram of $I_{n}^{*}$ for $n=3$ and 4 was motivated. In this paper we have discussed the cardinality for $1 \leq n \leq 10$, the Möbius function of and the Hasse diagram $1 \leq n \leq 6$.

\subsection{The Pointed Integer Partition for $n=1$}

The pointed integer partition for $n=1$ denoted by $1 \underline{0}$, and its set of pointed integer partition $I_{1}^{*}=\{\underline{1}, \underline{1}\}$. Thus $I_{1}^{*}$ has two pointed integer partitions and Figure 2 its Hasse diagram.

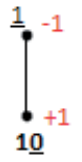

Fig. 2: The Hasse Diagram of $I_{1}^{\bullet}$

Thus the Möbius function for $I_{1}^{\bullet}$ recursively as follow:

$$
\begin{aligned}
& \mu(\underline{10})=\mu(\hat{0}, \underline{1} \underline{0})=\mu(\hat{0}, \hat{0})=1 \\
& \mu(\underline{1})=\mu(\hat{0}, \underline{1})=-\mu(\hat{0}, \hat{0})=-1
\end{aligned}
$$

Therefore $\mu\left(I_{1}^{*}\right)=-1=(-1)^{1}$.

\subsection{The Pointed Integer Partition for $\mathbf{n}=\mathbf{2}$}

The pointed integer partition for $n=2$ denoted by $11 \underline{0}$, to find the set of its pointed integer partition $I_{2}^{*}$, let us list all the covers of $11 \underline{0}$

$11 \underline{0} \preceq 2 \underline{0}, 1 \underline{1}, \quad 2 \underline{0} \preceq \underline{2}$ and $1 \underline{1} \preceq \underline{2}$.

Therefore, $I_{2}^{\bullet}=\{11 \underline{0}, 2 \underline{0}, 1 \underline{1}, \underline{2}\}$

Hence $I_{2}^{\cdot}$ has four pointed integer partitions, and its Hasse diagram is

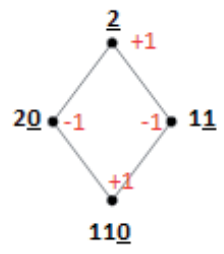

Fig. 3: The Hasse Diagram of $I_{2}^{\bullet}$.
The Möbius function for $I_{2}^{\bullet}$ recursively as follow:

$$
\begin{aligned}
& \mu(11 \underline{0})=\mu(\hat{0}, 11 \underline{0})=\mu(\hat{0}, \hat{0})=1 \\
& \mu(2 \underline{0})=\mu(\hat{0}, 2 \underline{0})=\mu(1 \underline{1})=\mu(\hat{0}, 1 \underline{1})=-\mu(\hat{0}, 11 \underline{0})=-1 \\
& \mu(\underline{2})=\mu(\hat{0}, \underline{2})=-\mu(\hat{0}, 11 \underline{0})-\mu(\hat{0}, 2 \underline{0})-(\hat{0}, 1 \underline{1})=1
\end{aligned}
$$

Therefore $\mu\left(I_{2}^{*}\right)=1=(-1)^{2}$

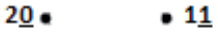

Fig. 4: The Hasse Diagram of $I_{2}^{\bullet}$ with $I_{n}^{\bullet} \backslash\{\hat{0}, \hat{1}\}$.

The figure 4 with the maximum and minimum elements removed. In this reduced poset, there is no greatest and least elements.

\subsection{The Pointed Integer Partition for $\mathbf{n}=3$}

The pointed integer partition for $n=3$ denoted by $111 \underline{0}$, to find the set of its pointed integer partition $I_{3}^{\cdot}$, let us list all the covers of $111 \underline{0}$

$111 \underline{0} \preceq 21 \underline{0}, 12 \underline{0}, 11 \underline{1}$. But $21 \underline{0}$ and $12 \underline{0}$ are the same pointed integer partition of $111 \underline{0}$, and they have the same covers, hence we can take one of them say $12 \underline{0}$, then

$$
\begin{array}{r}
12 \underline{0} \preceq 3 \underline{0}, 1 \underline{2}, 2 \underline{1} ; \quad 11 \underline{1} \preceq 2 \underline{1}, 1 \underline{2} \quad ; \quad 3 \underline{0} \preceq \underline{3} \\
2 \underline{1} \preceq \underline{3} \text { and } 1 \underline{2} \preceq \underline{3}
\end{array}
$$

Therefore, $I_{3}^{\cdot}=\{111 \underline{0}, 12 \underline{0}, 11 \underline{1}, 3 \underline{0}, 1 \underline{2}, 2 \underline{1}, \underline{3}\}$.

Thus $I_{3}^{*}$ has seven pointed integer partition and its Hasse diagram is [7].

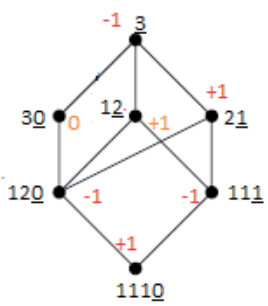

Fig. 5: The Hasse Diagram of $I_{3}^{\bullet}$.

The Möbius function for $I_{3}^{\bullet}$ recursively as follow:

$$
\begin{aligned}
& \mu(11 \underline{0})=\mu(\hat{0}, 111 \underline{0})=\mu(\hat{0}, \hat{0})=1 \\
& \mu(12 \underline{0})=\mu(\hat{0}, 12 \underline{0})=\mu(11 \underline{1})=-\mu(\hat{0}, 11 \underline{1})=-1 \\
& \mu(1 \underline{2})=\mu(\hat{0}, 1 \underline{2})=\mu(11 \underline{1})=-\mu(\hat{0}, 111 \underline{0})-\mu(\hat{0}, 12 \underline{0})- \\
& -\mu(\hat{0}, 11 \underline{1})=1 \\
& \mu(2 \underline{1})=\mu(\hat{0}, 2 \underline{1})=-\mu(\hat{0}, 111 \underline{0})-\mu(\hat{0}, 12 \underline{0})- \\
& -\mu(\hat{0}, 11 \underline{1})=1
\end{aligned}
$$




$$
\begin{aligned}
& \mu(\underline{3})=\mu(\hat{0}, \underline{3})=-\mu(\hat{0}, 111 \underline{0})-\mu(\hat{0}, 12 \underline{0})- \\
& \mu(\hat{0}, 11 \underline{1})-\mu(\hat{0}, \underline{3} \underline{0})-\mu(\hat{0}, 2 \underline{1})-\mu(\hat{0}, 1 \underline{2})=-1
\end{aligned}
$$

Therefore $\mu\left(I_{3}^{\bullet}\right)=-1=(-1)^{3}$

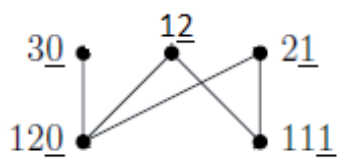

Fig. 6: The Hasse Diagram of $I_{3}^{\bullet}$ with no $\hat{0}$ and $\hat{1}$.

Figure 6 with the maximum and minimum elements removed. In this reduced poset, the top row of elements are all greatest elements, and the bottom row are all least elements, but there is no maximum and minimum element.

\subsection{The Pointed Integer Partition for $\mathrm{n}=4$}

The pointed integer partition for $n=4$ denoted by $1111 \underline{0}$, to find the set of its pointed integer partition $I_{4}^{\bullet}$, let us list all the covers of $1111 \underline{0}$

$1111 \underline{0} \preceq 211 \underline{0}, 121 \underline{0}, 112 \underline{0}, 111 \underline{1}$

But $211 \underline{0}, 121 \underline{0}$ and $112 \underline{0}$ are the same pointed integer partition of $1111 \underline{0}$, and the covers of one of them contains the covers of the others, thus let us take $1112 \underline{0}$

$112 \underline{0} \preceq 22 \underline{0}, 13 \underline{0}, 31 \underline{0}, 11 \underline{2}, 12 \underline{1}$, thus $13 \underline{0}$ and $31 \underline{0}$ are the same pointed integer partition of $112 \underline{\underline{0}}$ and they have the same cover, so we can take one of the two say $13 \underline{\underline{0}}$. So

$$
\begin{aligned}
13 \underline{0} & \preceq 4 \underline{0}, 3 \underline{1}, 1 \underline{3} \\
12 \underline{1} & \preceq 3 \underline{1}, 1 \underline{3}, 2 \underline{2} \\
22 \underline{0} & \preceq 4 \underline{0}, 2 \underline{2} \\
11 \underline{2} & \preceq 2 \underline{2}, 1 \underline{3} \\
4 \underline{0} & \preceq \\
3 \underline{1} & \preceq \\
2 \underline{2} & \preceq \\
1 \underline{3} & \preceq
\end{aligned}
$$

Therefore, for $n=4$,

$$
I_{4}^{\cdot}=\left\{\begin{array}{l}
1111 \underline{0}, 112 \underline{0}, 111 \underline{1}, 31 \underline{0}, 22 \underline{0}, \\
12 \underline{1}, 11 \underline{2}, 4 \underline{0}, 3 \underline{1}, 2 \underline{2}, 1 \underline{3}, \underline{4}
\end{array}\right\}
$$

Hence $I_{4}^{\bullet}$ has twelve pointed integer partitions, and figure 7 its Hasse diagram [8]

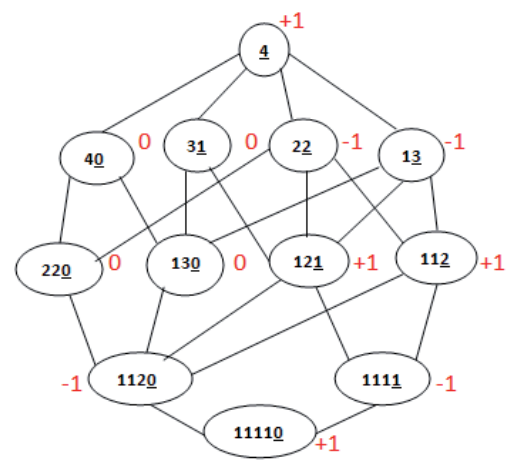

Fig. 7: The Hasse Diagram of $I_{4}^{\bullet}$

The Möbius function for $I_{4}^{\bullet}$ recursively as follow:

$$
\begin{aligned}
& \mu(1111 \underline{0})=\mu(\hat{0}, 1111 \underline{0})=\mu(\hat{0}, \hat{0})=1 \\
& \mu(112 \underline{0})=\mu(111 \underline{1})=\mu(\hat{0}, 112 \underline{0})=\mu(\hat{0}, 111 \underline{1})= \\
& =-\mu(\hat{0}, 111 \underline{0})=-1 \\
& \mu(13 \underline{0})=\mu(22 \underline{0})=\mu(\hat{0}, 13 \underline{0})=\mu(\hat{0}, 22 \underline{0})= \\
& =-\mu(\hat{0}, 1111 \underline{0})-\mu(\hat{0}, 112 \underline{0})=0 \\
& \mu(12 \underline{1})=\mu(11 \underline{2})=\mu(\hat{0}, 12 \underline{1})=\mu(\hat{0}, 11 \underline{2})= \\
& =-\mu(\hat{0}, 1111 \underline{0})-\mu(\hat{0}, 112 \underline{0})-\mu(\hat{0}, 111 \underline{1})=1 \\
& \mu(4 \underline{0})=\mu(\hat{0}, 4 \underline{0})=-\mu(\hat{0}, 1111 \underline{0})-\mu(\hat{0}, 112 \underline{0})- \\
& -\mu(\hat{0}, 22 \underline{0})-\mu(\hat{0}, 13 \underline{0})=0 \\
& \mu(3 \underline{1})=\mu(\hat{0}, 3 \underline{1})=-\mu(\hat{0}, 1111 \underline{0})-\mu(\hat{0}, 112 \underline{0})- \\
& -\mu(\hat{0}, 111 \underline{1})-\mu(\hat{0}, 13 \underline{0})-\mu(\hat{0}, 12 \underline{1})=0 \\
& \mu(2 \underline{2})=\mu(\hat{0}, 2 \underline{2})=-\mu(\hat{0}, 1111 \underline{0})-\mu(\hat{0}, 112 \underline{0})- \\
& -\mu(\hat{0}, 111 \underline{1})-\mu(\hat{0}, 11 \underline{2})-\mu(\hat{0}, 12 \underline{1})-\mu(\hat{0}, 22 \underline{0})=-1 \\
& \mu(1 \underline{3})=\mu(\hat{0}, 1 \underline{3})=-\mu(\hat{0}, 1111 \underline{0})-\mu(\hat{0}, 112 \underline{0})- \\
& -\mu(\hat{0}, 111 \underline{1})-\mu(\hat{0}, 13 \underline{0})-\mu(\hat{0}, 12 \underline{1})-\mu(\hat{0}, 11 \underline{2})=-1 \\
& \mu(\underline{4})=\mu(\hat{0}, \underline{4})=-\mu(\hat{0}, 111 \underline{0})-\mu(\hat{0}, 112 \underline{0})-\mu(\hat{0}, 111 \underline{1})- \\
& -\mu(\hat{0}, 13 \underline{0})-\mu(\hat{0}, 12 \underline{1})-\mu(\hat{0}, 22 \underline{0})-\mu(\hat{0}, 11 \underline{2})- \\
& -\mu(\hat{0}, 4 \underline{0})-\mu(\hat{0}, \underline{3} \underline{1})-\mu(\hat{0}, \underline{3})-\mu(\hat{0}, 2 \underline{2})=1 \\
& -
\end{aligned}
$$

Therefore $\mu\left(I_{4}^{*}\right)=1=(-1)^{4}$ 


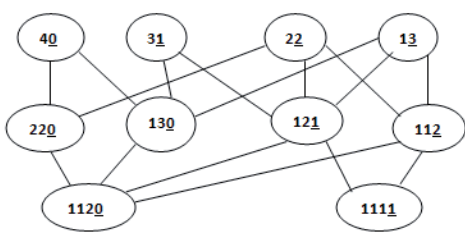

Fig. 8: The Hasse Diagram of $I_{4}^{\cdot}$ with no $\hat{0}$ and $\hat{1}$

A Figure 8 below when the maximum and minimum elements are removed. In this reduced poset, the top row of elements are all greatest elements, and the bottom row are all least elements, but there is no maximum and minimum element.

The set of pointed integer partition for $n=5$ with $11111 \underline{0}$ as a base element is denoted by $I_{5}^{*}$. List of all the covers of 111110 are

$11111 \underline{0} \preceq 2111 \underline{0}, 1211 \underline{0}, 1121 \underline{0}, 1112 \underline{0}, 1111 \underline{1}$.

But the partition 1112 $\underline{0}, 2111 \underline{0}, 1211 \underline{0}$ and 1121므, are the same pointed integer partition of $11111 \underline{0}$ and they have the same covers, so the covers of one of them contains all the covers of the others, thus let us take $1112 \underline{0}$, and hence

$1112 \underline{0} \preceq 212 \underline{0}, 122 \underline{0}, 113 \underline{0}, 311 \underline{0}, 131 \underline{0}, 1112,112 \underline{1}$

with the same manner to the above reason, let us take $212 \underline{0}$ from $212 \underline{0}$ and $122 \underline{0}$, and $113 \underline{0}$ from $113 \underline{0}, 311 \underline{0}$ and $131 \underline{0}$, so

$$
\begin{aligned}
& 212 \underline{0} \preceq 32 \underline{0}, 23 \underline{0}, 41 \underline{0}, 14 \underline{0}, 21 \underline{2}, 12 \underline{2}, 22 \underline{1} \\
& 113 \underline{0} \preceq 23 \underline{0}, 14 \underline{0}, 41 \underline{0}, 11 \underline{3}, 13 \underline{1} \\
& 111 \underline{2} \preceq 21 \underline{2}, 12 \underline{2}, 11 \underline{3} \\
& 112 \underline{1} \preceq 22 \underline{1}, 13 \underline{1}, 31 \underline{1}, 11 \underline{3}, 12 \underline{2}
\end{aligned}
$$

From equation (3) to (6) we have to take only the

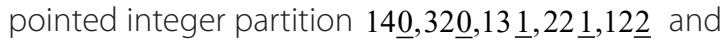
$11 \underline{3}$, since the remaining pointed integer partition are the same as to these partitions.

$$
\begin{aligned}
& 32 \underline{0} \preceq 5 \underline{0}, 3 \underline{2}, 2 \underline{3} \\
& 14 \underline{0} \preceq 5 \underline{0}, 1 \underline{4}, 4 \underline{1} \\
& 13 \underline{1} \preceq 4 \underline{1}, 1 \underline{4}, 3 \underline{2} \\
& 22 \underline{1} \preceq 4 \underline{1}, 2 \underline{3} \\
& 12 \underline{2} \preceq 3 \underline{2}, 1 \underline{4}, 2 \underline{3} \\
& 11 \underline{3} \preceq 2 \underline{3}, 1 \underline{4} \\
& 5 \underline{0} \preceq \underline{5} \\
& 4 \underline{1} \preceq \underline{5} \\
& 3 \underline{2} \preceq \underline{5} \\
& 2 \underline{3} \preceq \underline{5} \\
& 1 \underline{4} \preceq \underline{5}
\end{aligned}
$$

Therefor, for $n=5$,
$I_{5}^{\cdot}=\left\{\begin{array}{l}11111 \underline{0}, 1112 \underline{0}, 1111 \underline{1}, 212 \underline{0}, 113 \underline{0}, 121 \underline{1}, 111 \underline{2} \\ 14 \underline{0}, 32 \underline{0}, 13 \underline{1}, 22 \underline{1}, 12 \underline{2}, 11 \underline{3}, 5 \underline{0}, 4 \underline{1}, 3 \underline{2}, 2 \underline{3}, 1 \underline{4}, \underline{5}\end{array}\right\}$

Hence $I_{5}^{*}$ has nineteen pointed integer partitions, Figure 9 is its Hasse diagram. Similarly, the Möbius function for $I_{5}^{\cdot}$ computed recursively as above and $\mu\left(I_{5}^{\bullet}\right)=-1=(-1)^{5}$.

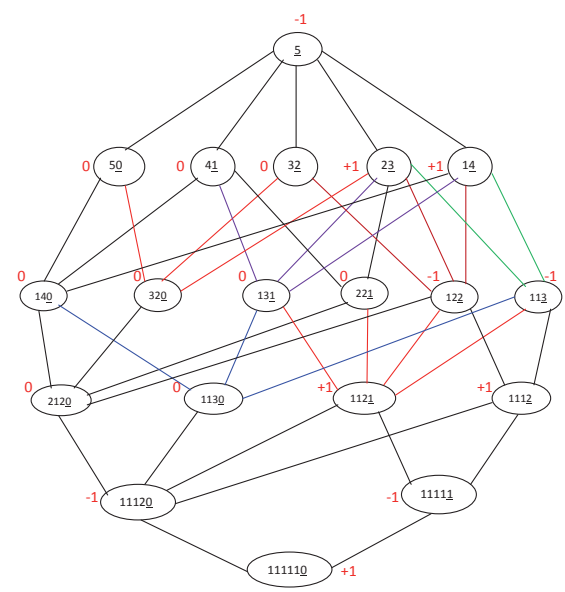

Fig. 9: The Hasse Diagram of $I_{5}^{\bullet}$

Figure 10 below is when the maximum and minimum elements are removed. In this reduced poset, the top row of elements are all greatest elements, and the bottom row are all least elements, but there is no maximum and minimum element.

The pointed integer partition for $n=6$ is denoted by $I_{6}^{*}$. To list all the covers of $111111 \underline{0}$ :

$$
\begin{gathered}
111111 \underline{0} \preceq 21111 \underline{0}, 12111 \underline{0}, 11211 \underline{0}, 11121 \underline{0}, \\
11112 \underline{0}, 11111 \underline{1}
\end{gathered}
$$

But the partitions 211110,121110,112110,111210 and $11112 \underline{0}$, are the same pointed integer partitions of $111111 \underline{0}$, so the covers of one of them contains the covers of the others, from those let us take 111120 .

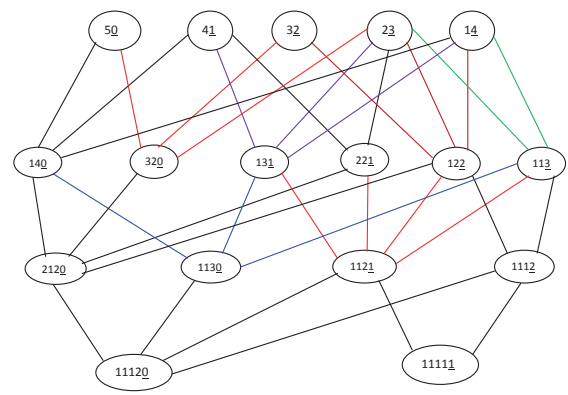

Fig. 10: The Hasse Diagram of $I_{5}^{\bullet}$ with no $\hat{0}$ and $\hat{1}$ 
Therefore, by the same techniques of $I_{1}^{*}$ to $I_{5}^{*}$ above

$$
\begin{aligned}
& 11112 \underline{0} \preceq 1122 \underline{0}, 1113 \underline{0}, 1112 \underline{1}, 1111 \underline{2} \\
& 1122 \underline{0} \preceq 222 \underline{0}, 132 \underline{0}, 114 \underline{0}, 112 \underline{2}, 112 \underline{1} \\
& 1113 \underline{0} \preceq 123 \underline{0}, 114 \underline{0}, 113 \underline{1}, 111 \underline{\underline{3}} \\
& 1112 \underline{1} \preceq 212 \underline{1}, 113 \underline{1}, 112 \underline{2}, 1113 \\
& 1111 \underline{2} \preceq 211 \underline{2}, 111 \underline{3} \\
& 222 \underline{0} \preceq 42 \underline{0}, 22 \underline{2} \\
& 123 \underline{0} \preceq 42 \underline{0}, 15 \underline{0}, 13 \underline{2}, 12 \underline{3}, 33 \underline{0}, 23 \underline{1} \\
& 114 \underline{0} \preceq 24 \underline{0}, 15 \underline{0}, 11 \underline{4}, 14 \underline{1} \\
& 212 \underline{1} \preceq 32 \underline{1}, 21 \underline{3}, 22 \underline{2}, 14 \underline{1} \\
& 113 \underline{1} \preceq 23 \underline{1}, 14 \underline{1}, 11 \underline{4}, 13 \underline{2} \\
& 112 \underline{2} \preceq 22 \underline{2}, 13 \underline{2}, 12 \underline{3}, 11 \underline{4} \\
& 121 \underline{2} \preceq 31 \underline{2}, 22 \underline{2}, 21 \underline{3}, 11 \underline{4} \\
& 111 \underline{3} \preceq 21 \underline{3}, 11 \underline{4} \\
& 42 \underline{0} \preceq 6 \underline{0}, 4 \underline{2}, 2 \underline{4} \\
& 33 \underline{0} \preceq 6 \underline{0}, 3 \underline{3} \\
& 15 \underline{0} \preceq 6 \underline{0}, 1 \underline{\underline{3}}, 5 \underline{1} \\
& 32 \underline{1} \preceq 5 \underline{1}, 2 \underline{4}, 3 \underline{\underline{3}} \\
& 14 \underline{1} \preceq 5 \underline{1}, 1 \underline{5}, 4 \underline{2} \\
& 13 \underline{2} \preceq 4 \underline{2}, 1 \underline{5}, 3 \underline{3} \\
& 22 \underline{2} \preceq 4 \underline{2}, 2 \underline{4} \\
& 21 \underline{3} \preceq 3 \underline{3}, 2 \underline{4}, 1 \underline{5} \\
& 11 \underline{4} \preceq 2 \underline{4}, 1 \underline{5} \\
& 6 \underline{0} \preceq \underline{6} \\
& 5 \underline{1} \preceq \underline{6} \\
& 4 \underline{2} \preceq \underline{6} \\
& 3 \underline{3} \preceq \underline{6} \\
& 2 \underline{4} \preceq \underline{6} \\
& 1 \underline{5} \preceq \underline{6}
\end{aligned}
$$

Hence for, $n=6$,

$I_{6}=\left\{\begin{array}{l}111111 \underline{0}, 11112 \underline{0}, 11111 \underline{1}, 1122 \underline{0}, 1113 \underline{0}, 1112 \underline{1}, \\ 1111 \underline{2}, 114 \underline{0}, 222 \underline{0}, 123 \underline{0}, 212 \underline{1}, 113 \underline{1}, 112 \underline{2}, 111 \underline{3}, \\ 42 \underline{0}, 33 \underline{0}, 15 \underline{0}, 32 \underline{1}, 14 \underline{1}, 13 \underline{2}, 22 \underline{2}, 21 \underline{3}, 11 \underline{4}, 6 \underline{0}, 5 \underline{1}, \\ 4 \underline{2}, 3 \underline{3}, 2 \underline{4}, 1 \underline{5}, \underline{6}\end{array}\right\}$

So $I_{6}^{*}$ has thirty pointed integer partitions and figure 11 is its the Hasse diagram with Möbius function.

Figure 12 is when the maximum and minimum elements are removed. In this reduced poset, the top row of elements are all greatest elements, and the bottom row are all least elements, but there is no unique maximum and minimum element.

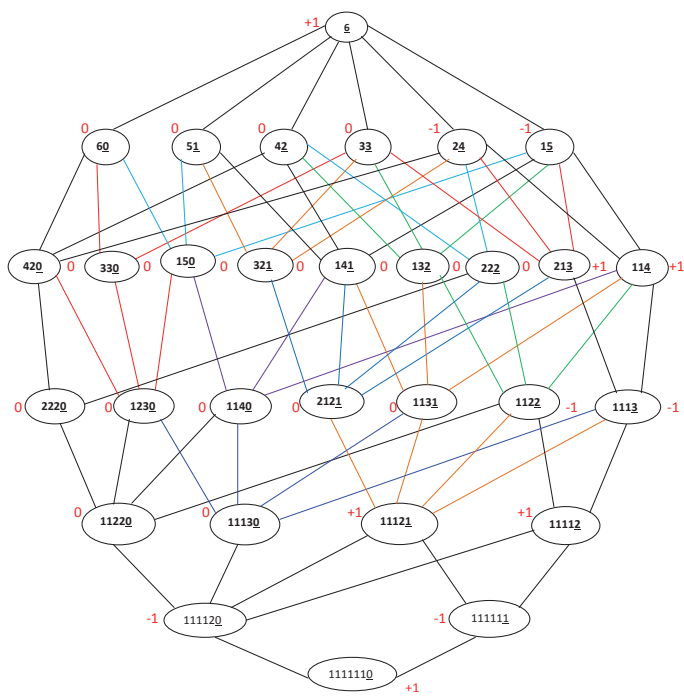

Fig. 11: The Hasse Diagram of $I_{6}^{\bullet}$

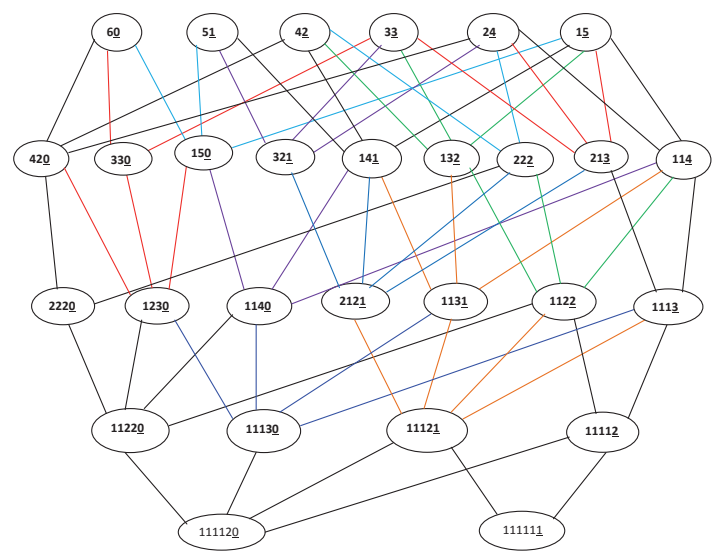

Fig. 12: The Hasse Diagram of $I_{6}^{\bullet}$ with no $\hat{0}$ and $\hat{1}$

Theorem 2.5.1. [Samuel and Melkamu] For $n \geq 1$, we have $\mu\left(I_{n}^{*}\right)=(-1)^{n}$, where $I_{n}^{*}$ is the set of all integer partition of a non-negative integer $n$, (see the proof in [8])

By using Definition (2.1.1) and similar manner to subsection from above the set of pointed integer partition and its cardinality for $7 \leq n \leq 10$ as follows:

$$
\begin{aligned}
& I_{7}^{\cdot}=\left\{\begin{array}{l}
1111111 \underline{0}, 111112 \underline{0}, 111111 \underline{1}, 11122 \underline{0}, 11113 \underline{0}, \\
11111 \underline{2}, 11112 \underline{1}, 1114 \underline{0}, 1123 \underline{0}, 1111 \underline{\underline{3}}, 1113 \underline{1}, \\
1222 \underline{0}, 1112 \underline{2}, 1122 \underline{1}, 115 \underline{0}, 124 \underline{0}, 111 \underline{4}, 114 \underline{1}, \\
133 \underline{0}, 223 \underline{0}, 112 \underline{3}, 113 \underline{2}, 123 \underline{1}, 122 \underline{2}, 222 \underline{1}, 16 \underline{0}, \\
25 \underline{0}, 11 \underline{5}, 15 \underline{1}, 34 \underline{0}, 12 \underline{4}, 14 \underline{2}, 24 \underline{1}, 13 \underline{3}, 33 \underline{1}, 22 \underline{3}, \\
23 \underline{2}, 7 \underline{0}, 1 \underline{6}, 6 \underline{1}, 2 \underline{5}, 5 \underline{2}, 3 \underline{4}, 4 \underline{3}, \underline{7}
\end{array}\right\} \\
& \left|I_{7}\right|=45
\end{aligned}
$$




$$
\begin{aligned}
I_{8}^{\cdot} & =\left\{\begin{array}{l}
11111111 \underline{0}, 1111112 \underline{0}, 1111111 \underline{1}, 11112 \underline{0}, \\
111113 \underline{\underline{0}}, 11111 \underline{2}, 111112 \underline{1}, 1111 \underline{0}, 11123 \underline{0}, \\
11111 \underline{\underline{3}}, 11113 \underline{1}, 11222 \underline{0}, 11112 \underline{2}, 11122 \underline{1}, \\
1115 \underline{0}, 1124 \underline{0}, 1111 \underline{4}, 1114 \underline{1}, 1133 \underline{0}, 1223 \underline{0}, \\
1112 \underline{\underline{3}}, 1113 \underline{2}, 2222 \underline{0}, 1123 \underline{1}, 1122 \underline{2}, 1222 \underline{1}, \\
116 \underline{0}, 125 \underline{0}, 111 \underline{5}, 1151,134 \underline{0}, 224 \underline{0}, 112 \underline{4}, 114 \underline{2}, \\
124 \underline{1}, 233 \underline{0}, 113 \underline{3}, 133 \underline{1}, 122 \underline{3}, 123 \underline{2}, 223 \underline{1}, 222 \underline{2}, \\
17 \underline{0}, 26 \underline{0}, 11 \underline{6}, 16 \underline{1}, 35 \underline{0}, 12 \underline{5}, 25 \underline{1}, 15 \underline{2}, 44 \underline{0}, 34 \underline{1}, \\
14 \underline{3}, 13 \underline{4}, 22 \underline{4}, 24 \underline{2}, 23 \underline{3}, 33 \underline{2}, 8 \underline{0}, 1 \underline{7}, 7 \underline{1}, 2 \underline{6}, 6 \underline{2}, 5 \underline{3}, \\
4 \underline{4}, 3 \underline{5}, \underline{8}
\end{array}\right\} \\
\left|I_{8}^{\cdot}\right| & =67
\end{aligned}
$$

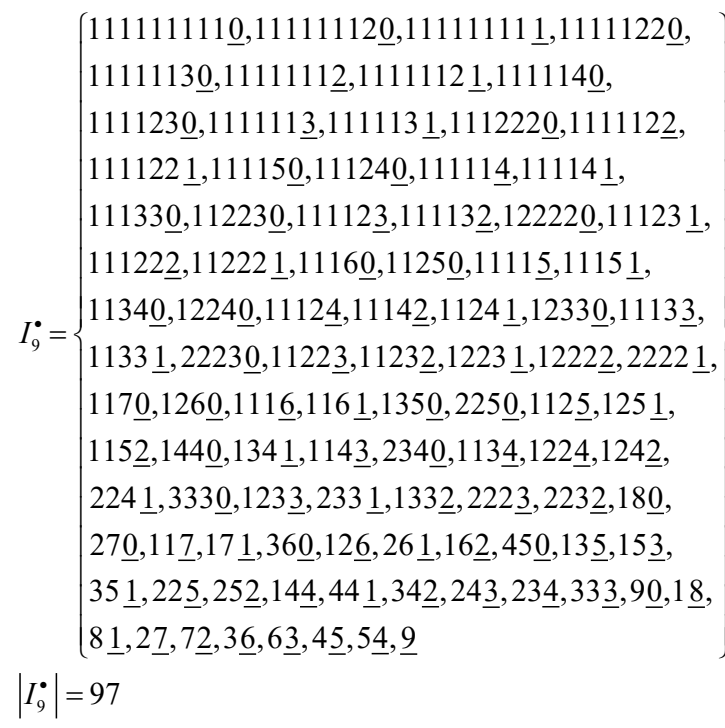

From subsections 2.2 to 2.8 we can conclude that,

(i) The Cardinality of $I_{n}^{\bullet}$ for $1 \leq n \leq 10$ are

$$
\begin{aligned}
\left|I_{1}^{\bullet}\right| & =2 & & \left|I_{6}^{\bullet}\right|=30 \\
\left|I_{2}^{\bullet}\right| & =4 & & \left|I_{7}^{\bullet}\right|=45 \\
\left|I_{3}^{\bullet}\right| & =7 & & \left|I_{8}^{\bullet}\right|=67 \\
\left|I_{4}^{\bullet}\right| & =12 & & \left|I_{9}^{\bullet}\right|=97 \\
\left|I_{5}^{\bullet}\right| & =19 & & \left|I_{10}^{\bullet}\right|=141 \\
\cdots & =\cdots & & \\
\cdots & =\cdots & & \\
\left|I_{n}^{\bullet}\right| & =? & &
\end{aligned}
$$

(ii) There are $2 n$ numbers of pointed integer partition whose Möbius number different from zero.

\section{Shellability of Pointed Integer Partition} 3.1 Lexicographic Shellability

There are two basic versions of lexicographic

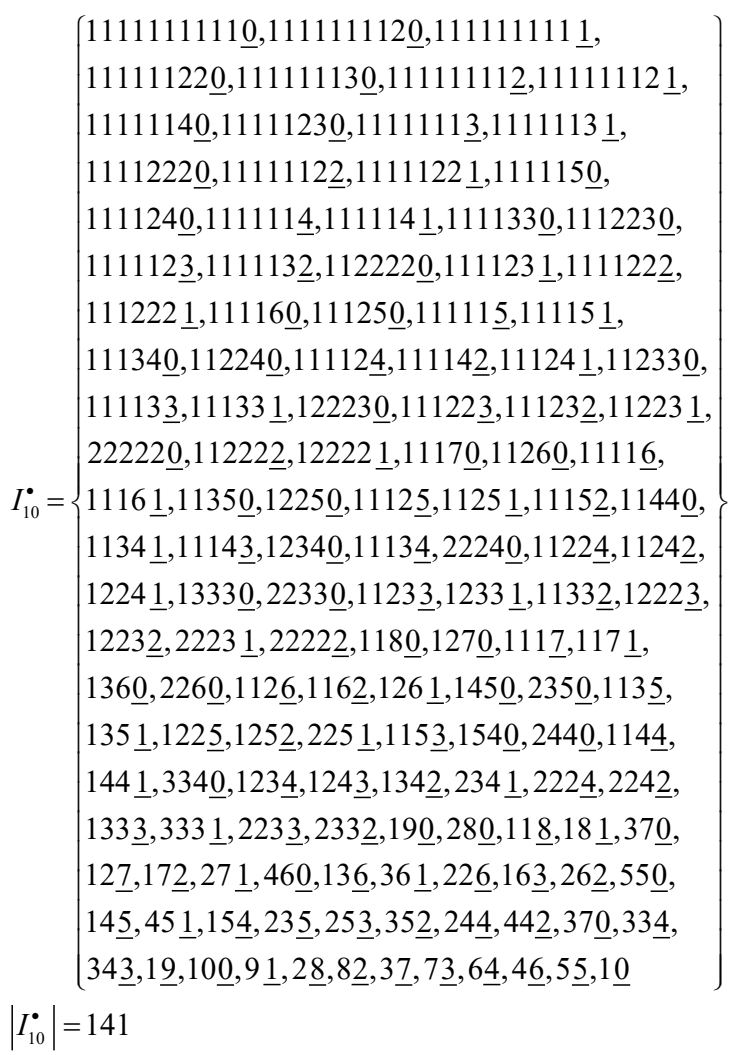

shellability, EL-shellability and CL-shellability. In this paper we will see EL-shellability, and discuss some of its consequences.

If $x$ and $y$ are elements in a poset $P$, we say that $y$ covers $x$ when $x<y$ but there is no $z \in P$, so that $x<z<y$. In this situation, we write $x \lessdot y$, and may also say that $x \lessdot y$ is a cover relation. Thus, a cover relation is an edge in the Hasse diagram of $P$. A rooted cover relation is a cover relation $x \lessdot y$ together with a maximal chain from $\hat{0}$ to $x$ (called the root) [9].

An edge labeling of a bounded poset $P$ is a map $\lambda: \varepsilon(P) \rightarrow \wedge$, where $\varepsilon(P)$ is the set of edges of the Hasse diagram of $P$, i.e., the covering relations $x \lessdot y$ of $P$, and $\wedge$ is some poset (usually the integers $\mathbb{Z}$ with its natural total order relation). Given an edge labeling $\lambda: \varepsilon(P) \rightarrow \wedge$, one can associate a word $\lambda(c)=\lambda\left(\hat{0}, x_{1}\right) \lambda\left(x_{1}, x_{2}\right) \cdots \lambda\left(x_{t}, \hat{1}\right)$ with each maximal chain $c=\left(\hat{0} \lessdot x_{1} \lessdot \cdots \lessdot x_{t} \lessdot \hat{1}\right)$. We say that $c$ is increasing if the associated word $\lambda(c)$ is strictly increasing. That is, $c$ is increasing if $\lambda\left(\hat{0}, x_{1}\right)<\lambda\left(x_{1}, x_{2}\right)<\cdots<\lambda\left(x_{t}, \hat{1}\right)$.

We say that $c$ is decreasing if the associated word $\lambda(c)$ is weakly increasing. We can order the maximal 
chains lexicographically by using the lexicographic order on the corresponding words. Any edge labeling $\lambda$ of $P$ restricts to an edge labeling of any closed interval $[x, y]$ of $P$. So we may refer to increasing and decreasing maximal chains of $[x, y]$, and lexicographic order of maximal chains of $[x, y]$.

Definition 3.1.1. Let $P$ be a bounded poset. An edgelexicographical labeling (EL-labeling, for short) of $P$ is an edge labeling such that in each closed interval $[x, y]$ of $P$, there is a unique increasing maximal chain, which lexicographically precedes all other maximal chains of $[x, y][9]$.

An example of an EL-labeling of a poset is given in Figure 13. The leftmost chain, which has associated word 123, is the only increasing maximal chain of the interval $[\hat{0}, \hat{1}]$. It is also lexicographically less than all other maximal chains. One needs to check each interval to verify that the labeling is indeed an EL-labeling. A bounded poset that admits an ELlabeling is said to be edge-lexicographic shellable (EL-shellable, for short).

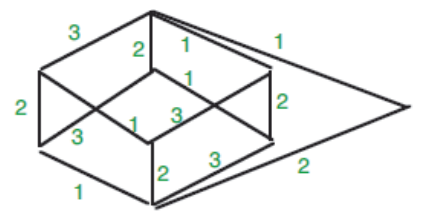

Fig. 13: EL-labeling

\subsection{EL-Shellability of $R_{n}$}

\section{Definition 3.2.1.}

(i) Let $n$ be a non-negative integer with $n \geq 3$, then $R_{n}$ is the set of pointed integer partitions with Möbius function either -1 or +1 .

(ii) Let $n$ be a non-negative integer with $n \geq 3$, then $I_{n}^{*}$ is set of all pointed integer partitions that has only zero Möbius function.

(iii) $I_{n}^{\bullet}$ denote the set of pointed integer partitions (see section 2.1).

(iii) For $n \geq 3$ and $\lambda \in \overline{I_{n}^{*}}=\left\{\tau \in I_{n}^{\bullet}: \tau \notin R_{n}\right\}$ then $\mu_{I_{i}^{*}}(\hat{0}, \lambda)=0$.

\section{Theorem 3.2.1.}

Let $R_{n}=\{1 \cdots 2 \underline{i}: 0 \leq i \leq n-1\} \bigcup\{1 \cdots 1 \underline{i}: 0 \leq i \leq n-1\} \bigcup$ $\{2 \underline{n-2}\} \bigcup\{\underline{n}\} \subseteq I_{n}^{\bullet}$. Then $\mu_{R_{n}}(\hat{0}, \hat{1})=(-1)^{n}[8]$.

Example 3.2.1. Consider $\mu\left(I_{3}^{*}\right)=-1=(-1)^{3}$ and $\mu\left(R_{3}\right)=-1=(-1)^{3}$ where $R_{3}=\{1 \cdots 2 \underline{i}: 0 \leq i \leq 2\} \bigcup$ $\{1 \cdots 1 \underline{i}: 0 \leq i \leq 2\} \cup\{2 \underline{1}\} \cup\{\underline{3}\}$, Figure 14 is its Hasse
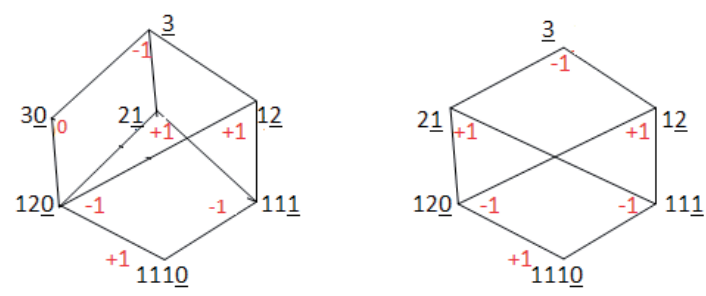

Fig. 14: The Hasse diagram of $I_{3}^{\bullet}$ and $R_{3}$

Example 3.2.2. Consider $\mu\left(I_{4}^{*}\right)=-1=(-1)^{4}$ and $\mu\left(R_{4}\right)=-1=(-1)^{4}$ where $R_{4}=\{1 \cdots 2 \underline{i}: 0 \leq i \leq 3\} \bigcup$ $\{1 \cdots 1 \underline{i}: 0 \leq i \leq 3\} \cup\{2 \underline{2}\} \cup\{\underline{4}\}$, Figure 15 is its Hasse

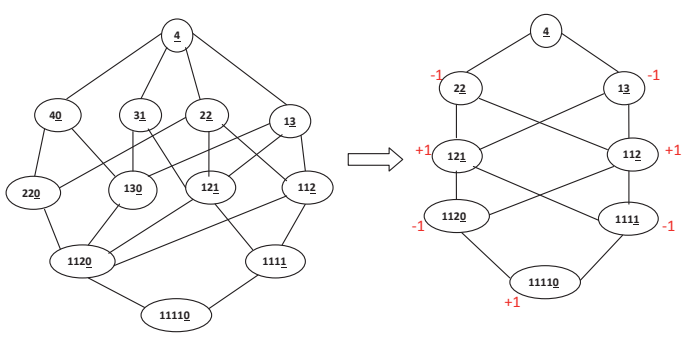

Fig. 15: The Hasse diagram of $I_{4}^{\bullet}$ and $R_{4}$

Proposition 3.2.1. If $\lambda \in \overline{I_{n}^{*}}$, then $\left\{\eta \in R_{n}: \eta \preceq \lambda\right\}$ has a unique maximal element [8].

For instance, consider

$I_{4}^{*}=\left\{\begin{array}{l}1111 \underline{0}, 112 \underline{0}, 111 \underline{1}, 13 \underline{0}, 22 \underline{0}, 12 \underline{1}, 11 \underline{2}, \\ 4 \underline{0}, 3 \underline{1}, 2 \underline{2}, 1 \underline{3}, \underline{4}\end{array}\right\}$.

It is easy to check that,

$\overline{I_{4}^{*}}=\{22 \underline{0}, 13 \underline{0}, 4 \underline{0}, 3 \underline{1}\}$

$R_{4}=\{111 \underline{10}, 11 \underline{0} \underline{0}, 111 \underline{1}, 12 \underline{1}, 11 \underline{2}, 2 \underline{2}, 1 \underline{3}, \underline{4}\}$.

Now, for $13 \underline{0} \in \overline{I_{4}^{*}}$,

$\left\{\eta \in R_{4}: \eta \preceq 13 \underline{0}\right\}=\{1111 \underline{0}, 112 \underline{0}\}=\left\{\eta \in R_{4}: \eta \preceq 22 \underline{0}\right\}=$ $=\left\{\eta \in R_{4}: \eta \preceq 4 \underline{0}\right\}$

For this set the unique maximal element is $112 \underline{0} \in R_{4}$. Consider $3 \underline{1} \in \overline{I_{4}^{*}}$, so that $\left\{\eta \in R_{4}: \eta \preceq 3 \underline{1}\right\}=$ $\{1111 \underline{0}, 112 \underline{0}, 111 \underline{1}, 12 \underline{1}\}$.

The unique maximal element for this set is $12 \underline{1}$.

\subsection{EL-Labeling of $R_{n}$}

[7] Verified the shellability of $R_{n}$ for $3 \leq n \leq 8$ by labeling, the right side edge set numbers from 1 through $n$ starting from the most bottom edge, and to assign the left side edge set assign numbers 1 to $n$ starting from the most top edge.

To assign edge set inclined to the left start labeling by assigning the number $n-1$ to the top most left inclined edge set and continue it till you assign 2 to an edge, those edge set inclined to the right start 
labeling by assigning the number $\frac{n}{2}($ if $n=4,6,8, \cdots)$ and $\frac{n+1}{2}($ if $n=3,5,7, \cdots)$ to the top most right inclined edge set and continue it till you assign 1 to an edge, after that you will remain either one edge or two edges unassigned based on whether $n$ is even or odd for which you can assign any number less than or equal to $\frac{n}{2}$ or $\frac{n+1}{2}$ respectively, and conjectured that $R_{n}$ admit an EL-labeling which is EL-shellable.

Thus, to prove the conjecture of [7], let us see the cardinality, set partition, Hasse diagram and the edge labeling for $3 \leq n \leq 11$.

$$
\begin{aligned}
& \text { For } n=3 \\
& R_{3}=\{111 \underline{0}, 12 \underline{0}, 11 \underline{1}, 1 \underline{2}, 2 \underline{1}, \underline{3}\} \\
& \left|R_{3}\right|=6 \\
& \text { For } n=4 \\
& R_{4}=\{1111 \underline{0}, 112 \underline{0}, 111 \underline{1}, 11 \underline{2}, 12 \underline{1}, 2 \underline{2}, 1 \underline{3}, \underline{4}\} \\
& \left|R_{4}\right|=8 \\
& \text { For } n=5 \\
& R_{5}=\left\{\begin{array}{l}
11111 \underline{0}, 1112 \underline{0}, 1111 \underline{1}, 121 \underline{1}, 111 \underline{2}, 21 \underline{2}, 11 \underline{3}, \\
2 \underline{3}, 1 \underline{4}, \underline{5}
\end{array}\right\} \\
& \left|R_{5}\right|=10 \\
& R_{6}=\left\{\begin{array}{l}
111111 \underline{0}, 11112 \underline{0}, 11111 \underline{1}, 1112 \underline{1}, 1111 \underline{2}, \\
112 \underline{2}, 111 \underline{3}, 12 \underline{3}, 11 \underline{4}, 2 \underline{4}, 1 \underline{5}, \underline{6}
\end{array}\right\} \\
& \left|R_{6}\right|=12
\end{aligned}
$$

For $n=7$

$$
\begin{aligned}
R_{7} & =\left\{\begin{array}{l}
1111111 \underline{0}, 111112 \underline{0}, 111111 \underline{1}, 11112 \underline{1}, \\
11111 \underline{2}, 1112 \underline{2}, 1111 \underline{3}, 112 \underline{3}, 111 \underline{4}, 12 \underline{4}, 11 \underline{\underline{5}}, \\
2 \underline{5}, 1 \underline{6}, \underline{7}
\end{array}\right\} \\
\left|R_{7}\right| & =14
\end{aligned}
$$

For $n=8$

$$
\begin{aligned}
R_{8} & =\left\{\begin{array}{l}
11111111 \underline{0}, 1111112 \underline{0}, 1111111 \underline{1}, 111112 \underline{1}, \\
111111 \underline{2}, 11112 \underline{2}, 11111 \underline{3}, 1112 \underline{3}, 1111 \underline{4}, \\
112 \underline{4}, 111 \underline{5}, 12 \underline{5}, 11 \underline{\underline{6}}, 2 \underline{6}, 1 \underline{7}, \underline{8}
\end{array}\right\} \\
\left|R_{8}\right| & =16
\end{aligned}
$$

For $n=9$

$$
\begin{aligned}
R_{9} & =\left\{\begin{array}{l}
111111111 \underline{0}, 11111112 \underline{0}, 11111111 \underline{1}, \\
1111112 \underline{1}, 1111111 \underline{2}, 111112 \underline{2}, 11111 \underline{\underline{3}}, \\
11112 \underline{\underline{3}}, 11111 \underline{4}, 1112 \underline{4}, 1111 \underline{5}, 112 \underline{5}, 111 \underline{6}, \\
12 \underline{6}, 11 \underline{7}, 2 \underline{7}, 1 \underline{8}, \underline{9}
\end{array}\right\} \\
\left|R_{9}\right| & =18
\end{aligned}
$$

For $n=10$

$$
\begin{aligned}
R_{10} & =\left\{\begin{array}{l}
111111111 \underline{0}, 111111112 \underline{0}, 111111111 \underline{1}, \\
11111112 \underline{1}, 11111111 \underline{2}, 1111112 \underline{2}, \\
1111111 \underline{3}, 111112 \underline{\underline{3}}, 111111 \underline{4}, 11112 \underline{4}, \\
11111 \underline{5}, 1112 \underline{5}, 1111 \underline{6}, 112 \underline{6}, 111 \underline{7}, 12 \underline{\underline{z}}, 1 \underline{8}, \\
1 \underline{9}, 2 \underline{8}, 1 \underline{0}
\end{array}\right\} \\
\left|R_{10}\right| & =20
\end{aligned}
$$

For $n=11$

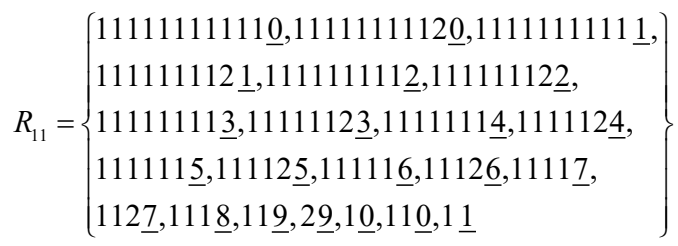$$
\left|R_{11}\right|=22
$$

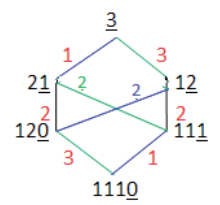

Fig. 16: The Hasse diagram of $R_{3}$

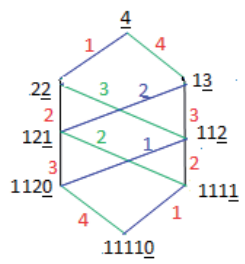

Fig. 17: The Hasse diagram of $R_{4}$

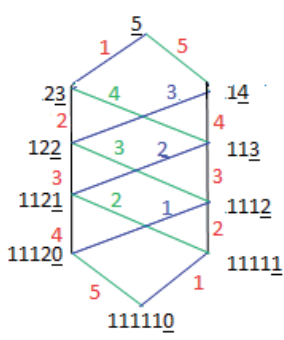

Fig. 18: The Hasse diagram of $R_{5}$

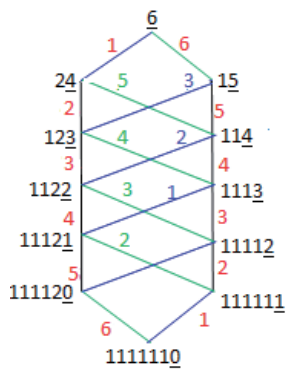

Fig. 19: The Hasse diagram of $R_{6}$ 


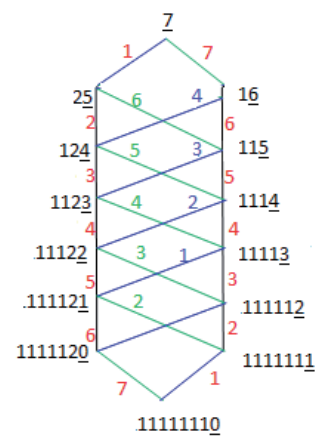

Fig. 20: The Hasse diagram of $R_{7}$

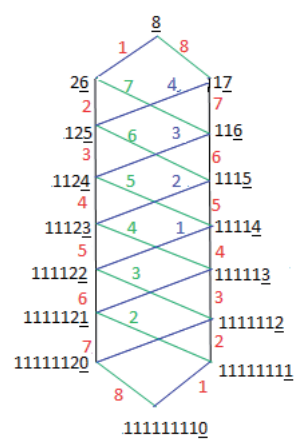

Fig. 21: The Hasse diagram of $R_{8}$

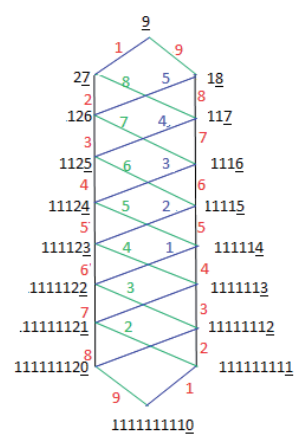

Fig. 22: The Hasse diagram of $R_{9}$

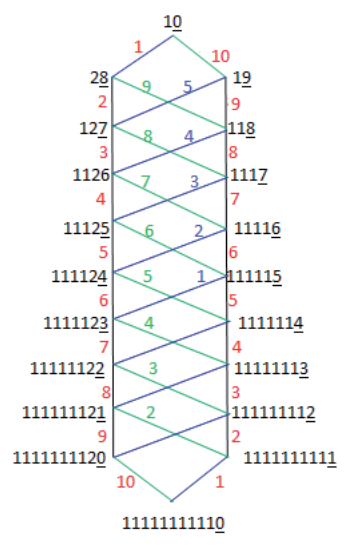

Fig. 23: The Hasse diagram of $R_{10}$

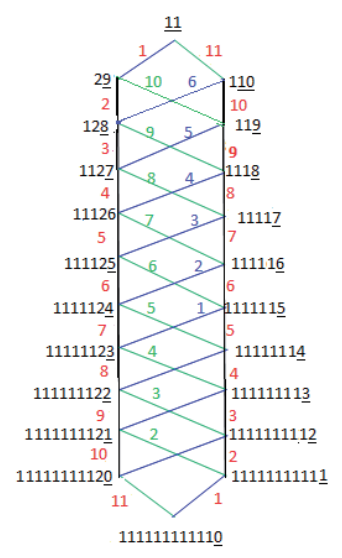

Fig. 24: The Hasse diagram of $R_{11}$

Theorem 3.3.1. The cardinality of $R n$ is equal to $2 n$, for $n \geq 1$.

Proof. Observation: Let $r_{n}=\left|R_{n}\right|$

From the Hasse diagram of previous sections 2.2, 2.3 and 3.3 we can observe that

$r_{1}=2$

$r_{2}=4$

$r_{3}=6$

$r_{4}=8$

$r_{5}=10$

$\cdots=\cdots$

$\cdots=\cdots$

$\cdots=\cdots$

$r_{n}=r_{n-1}+2$

$r_{n}=r_{n-1}+2$, for all non negative integer $\mathrm{n}, n \geq 1$

Let $n=1, r_{1}=r_{0}+2=2, \Rightarrow r_{0}=0$

In the recursive relation of the sequence $r_{n}=r_{n-1}+2$ Let $R(x)=\sum_{n \geq 0} r_{n} x^{n}$ be the generating function of $\left\{r_{n}\right\}_{n=0}^{\infty}$

$$
\begin{gathered}
\sum_{n \geq 1} r_{n} x^{n}=\sum_{n \geq 1} r_{n-1} x^{n}+2 \sum_{n \geq 1} x^{n} \\
R(x)-r_{0}=x \sum_{n \geq 0} r_{n-1} x^{n-1}+2\left(x\left(1+x+x^{2}+\cdots\right)\right) \\
R(x)=x R(x)+2\left(x\left(\frac{1}{1-x}\right)\right) \\
R(x)-x R(x)=2\left(x\left(\frac{1}{1-x}\right)\right) \\
R(x)=2\left(x\left(\frac{1}{(1-x)^{2}}\right)\right), \text { by partial fraction }
\end{gathered}
$$




$$
\begin{aligned}
\text { Let } R(x) & =\frac{2 x}{(1-x)^{2}}=\frac{A}{1-x}+\frac{B}{(1-x)^{2}} \\
\Rightarrow A(1-x)+B & =2 x \\
A & =-2 \text { and } B=2 \\
\Rightarrow \frac{2 x}{(1-x)^{2}} & =\frac{-2}{(1-x)}+\frac{2}{(1-x)^{2}} \\
\Rightarrow & =-2 \sum_{k \geq 0} x^{k}+2 \sum_{k \geq 0}\left(\begin{array}{c}
-2 \\
k
\end{array}\right) x^{k}(-1)^{k} \\
& =-2 \sum_{k \geq 0} x^{k}+2 \sum_{k \geq 0}\left(\begin{array}{c}
k-(-2)-1 \\
k
\end{array}\right) x^{k} \\
& =-2 \sum_{k \geq 0} x^{k}+2 \sum_{k \geq 0}\left(\begin{array}{c}
k+1 \\
k
\end{array}\right) x^{k} \\
& =-2 \sum_{k \geq 0} x^{k}+2 \sum_{k \geq 0}(k+1) x^{k} \\
& =2 \sum_{k \geq 0}(k+1-1) x^{k}
\end{aligned}
$$

$\left[x^{n}\right] R(x)=r_{n}=2 n$

Therefore, $\left|R_{n}\right|=2 n$

Hence, the edge labeling, the cardinality and the Hasse diagram of $R_{n}$ for $3 \leq n \leq 11$ has been seen above and based on the conjecture of [7] we have conclude the following:

(1) As [7] verified there is either one or two remaining unassigned right inclined edges based on whether $n$ is even or odd for which you can assign any number less than or equal to $\frac{n}{2}$ or $\frac{n+1}{2}$ respectively, thus we have got the new results on this verification.

i.e,

i. For $3 \leq n \leq 5$ their is no unassigned right inclined edge.

ii. For $n=6$ and 7 there is one unassigned right inclined edge.

iii. For $n=8$ and 9 there are two unassigned right inclined edges.

iv. For $n=10$ and 11 there are three unassigned right inclined edges.

(2) For $n=6$ and $n=7$ no need of assign the remaining right inclined unassigned edge by $\frac{n}{2}$ or $\frac{n+1}{2}$ based on whether $n$ is even or odd respectively to get maximum chain.

(3) For $n \geq 8$ by assigned 3 on the remaining unassigned right inclined edges between $1111 \cdots 121$ and $1111 \cdots 1 \underline{3}$ we got a maximum chain.

Conjecture. For $n \geq 8$ there needed to assign 3 on the remaining unassigned right in-clined edges of $R_{n}$ between $1111 \cdots 12 \underline{1}$ and $1111 \cdots 1 \underline{3}$ to got maximum chain, as verified in the above subsection 3.3 and the
Hasse diagram 21 to 24

Lemma 3.3.1. For all $n, n \geq 4,\left|R_{n}\right|=\left|R_{n-1}\right|+2$

Proof. Given $\lambda$ be a pointed integer partition of $n-1$ with Möbius number -1 and +1 Add $2 \underline{n-1}$ and $1 \underline{n-1}$ to the left and right top corner of the Hasse diagram of $\lambda$ respectively we get a pointed integer partition of $n$ with Möbius number of -1 and +1

$\Rightarrow\{\lambda, 2 \underline{n-2}, \underline{1 n-1}\} \in R_{n}$

since $\lambda$ is arbitrary, we have $R_{n}=R_{n-1}+2$

$\Rightarrow\left|R_{n}\right|=\left|R_{n-1}\right|+2$

Let $\operatorname{URIE}\left(R_{n}\right)$ denote unassigned inclined edge of $R_{n}$

Lemma 3.3.2. For all non negative integer $n, n \geq 6$

i) If $n$ is even, then $\left|\operatorname{URIE}\left(R_{n}\right)\right|=\left|\operatorname{URIE}\left(R_{n+1}\right)\right|$

ii) If $n$ is odd, then $\left|\operatorname{URIE}\left(R_{n+1}\right)\right|=\left|\operatorname{URIE}\left(R_{n}\right)\right|+1$

Proof. (i) Let $\lambda \in R_{n-1}$, where $n-1$ is even, then in the Hasse diagram of $\lambda$ the right inclined edges are assined from the top to the bottom by

$\frac{n-1}{2}, \frac{n-1}{2}-1, \frac{n-1}{2}-2, \cdots 1$
i.e. $\frac{n-1}{2}, \frac{n-3}{2}, \frac{n-5}{2}, \cdots 1$

from Lemma 3.1, we have shown that we can get a pointed integer partition of $n$ with Möbius number -1 and +1 by adding $2 \underline{n-2}$ to the left corner and $\underline{n-1}$ to the right corner of the Hasse diagram of $\lambda$.

In this case we have increased the number of right inclined edge by 1 at the top and these edges are labeled by

$\frac{n+1}{2}, \frac{n+1}{2}-1, \frac{n+1}{2}-2, \cdots 1$

i.e. $\frac{n+1}{2}, \frac{n-1}{2}, \frac{n-3}{2}, \cdots 1$

$\Rightarrow$ The number of URIE of $\lambda$ and $\{\lambda, 2 \underline{n-2}, \underline{1 n-1}\}$

are equal.

$\Rightarrow$ For all odd natural number $n, n \geq 7$, we have $\left|\operatorname{URIE}\left(R_{n-1}\right)\right|=\left|\operatorname{URIE}\left(R_{n}\right)\right|$

$\Rightarrow\left|\operatorname{URIE}\left(R_{n}\right)\right|=\left|\operatorname{URIE}\left(R_{n+1}\right)\right|$

Proof. (ii) $\lambda \in R_{n}$, where $\mathrm{n}$ is odd

In the Hasse digram of $\lambda$, the right inclined edges are assigned from top bottom by

$\frac{n+1}{2}, \frac{n+1}{2}-1, \frac{n+1}{2}-2, \cdots 1$

i.e. $\frac{n+1}{2}, \frac{n-1}{2}, \frac{n-3}{2}, \cdots 1$

From Lemma 3.3.1 we have shown that we can get a pointed integer partition of $\mathrm{n}$ with Möbius number -1 and +1 by adding $2 n-2$ to the left 
corner and $\underline{1 n-1}$ to the right corner of the Hasse diagram of $\lambda$.

In this case we have one additional right inclined edge at the top, since $n+1$ is even these edges are labelled by

$$
\begin{aligned}
& \frac{n+1}{2}, \frac{n+1}{2}-1, \frac{n+1}{2}-2, \cdots 1 \\
& \text { i.e. } \frac{n+1}{2}, \frac{n-1}{2}, \frac{n-3}{2}, \cdots 1
\end{aligned}
$$

since we have one extra inclined edge in $\{\lambda, 2 \underline{n-2}, 1 \underline{n-1}\}$, so the number of UREI of $\{\lambda, 2 n-2,1 n-1\}$ is more than $\lambda$ by 1

$$
\Rightarrow\left|\overline{\operatorname{URIE}}\left(\overline{R_{n+1}}\right)\right|=\left|\operatorname{URIE}\left(R_{n}\right)\right|+1
$$

Theorem 3.3.2. For all non-negative integer $n, n \geq 6$ the cardinality of unassigned right inclined edge of

$$
R_{n}= \begin{cases}\frac{n-4}{2}, & \text { if } n \text { is even } \\ \frac{n-5}{2}, & \text { if } n \text { is odd }\end{cases}
$$

Proof. Prove by induction

For $n=6$, we have shown in the Hasse diagram 19 i.e $\left|\operatorname{URIE}\left(R_{n}\right)\right|=1=\frac{6-4}{2}=\frac{2}{2}=1$,

it is true

Assume that the assertion of the theorem is true for all $\mathrm{k}, 6 \leq k \leq n$

We want to show it is true for $n+1$

Case 1. If $n$ is even

In this case, we have show in Lemma 3.3.2 the

$\left|\operatorname{URIE}\left(R_{n}\right)\right|=\left|\operatorname{URIE}\left(R_{n+1}\right)\right|$

By induction hypothesis, we have

$\left|\operatorname{URIE}\left(R_{n+1}\right)\right|=\left|\operatorname{URIE}\left(R_{n}\right)\right|=\frac{n-4}{2}=\frac{n+1-5}{2}$

Case 2. If $n$ is even

$\left|\operatorname{URIE}\left(R_{n+1}\right)\right|=\left|\operatorname{URIE}\left(R_{n}\right)+1\right|=\frac{n-5}{2}+1=\frac{n-5+2}{2}=$

$=\frac{n-3}{2}=\frac{n+1-4}{2}$

Therefore,

$$
R_{n}= \begin{cases}\frac{n-4}{2}, & \text { if } n \text { is even } \\ \frac{n-5}{2}, & \text { if } n \text { is odd }\end{cases}
$$

In general based on [7], Hasse diagrams 21 to 24, conjecture above, Lemma 3.3.1 and 3.3.2 and theorem 3.3.2 we can conclude that $R_{n}$ admits EL-labeling which is EL-shellable.

\section{Conclusion and Open Problems}

\subsection{Conclusions}

We began by reviewing some basic concepts of partial order set(poset), Hasse diagram, integer partition and set partition. Then we went to pointed integer partition by defining, listing the pointed integer partitions and the Hasse diagram based on their cover relation, thus we got the cardinality of pointed integer partition for $1 \leq n \leq$ 10 from those the pointed integer partitions where $n=1$ and $n=2$ are trivial and for $n=3$ and $n=4$ are studied by [8] the rest

$\mid I_{5} \cdot=19$

$\left|I_{6}^{\bullet}\right|=30$

$\left|I_{7}^{\bullet}\right|=45$

$\left|I_{8}^{\bullet}\right|=67$

$\left|I_{9}^{\bullet}\right|=97$

$\left|I_{10}\right|=141$ are new results.

While when we went to shellability of pointed integer partition, we have discussed the basic concepts of lexicographic shellability, EL-labeling, shellability, cardinality and the Hasse diagram of $R_{n}$ following the publication of [1], [3], [4] and [8], and again to verify and prove the conjecture of [7] we have defined $\overline{I_{n}^{*}}, R_{n}, \mu_{R n}$ and $\mu_{I_{n}^{*}}$ and new results done, i.e.

(i) To get maximum chain of $R_{n}$ for $n=6$ and $n=7$ no need of assign the remaining unassigned right inclined edges by $\frac{n}{2}$ or $\frac{n+1}{2}$ based on whether $n$ is even or odd respectively, but for $n \geq 8$ there needed to assign 3 on the remaining unassigned right inclined edges between $1111 \cdots 12 \underline{1}$ and $1111 \cdots 1 \underline{3}$.

(ii) For $3 \leq n \leq 5$ there is no unassigned right inclined edges.

(iii) For $n=6$ and 7 there is one unassigned right inclined edges.

(iv) For $n=8$ and 9 there are two unassigned right inclined edges.

(v) For $n=10$ and 11 there are three unassigned right inclined edges.

(vi) For all non-negative integer $\mathrm{n}, n \geq 6$ the cardinality of unassigned right inclined edge of

$R_{n}= \begin{cases}\frac{n-4}{2}, & \text { if } n \text { is even } \\ \frac{n-5}{2}, & \text { if } n \text { is odd }\end{cases}$ 
(vii) The cardinality of $R_{n}$ is equal to $2 n$, for $n \geq 1$

\subsection{Open Problems}

1) Is there recursive formula for $I_{n}^{\bullet}$ where $n \geq 1$ ?

2) Is there a relationship between the Hasse diagrams of $I_{n}^{\bullet}$ with no $\hat{0}$ and $\hat{1}$ with respect to theory of graph?

3.) Prove

i) For $n \geq 8$ by assigning 3 on the remaining unassigned right inclined edges of $R_{n}$ between $1111 \cdots 12 \underline{1}$ and $1111 \cdots 1 \underline{3}$ we have got maximum chain.

ii) To get maximum chain of $R_{n}$ for $n=6$ and $n=7$ no need of assign the remaining unassigned right inclined edges.

\section{Acknowledgments}

The first author would like to thank Dr. Tomas Vetrik for hosting him at his institution and providing guidance while he was working on this project. He also acknowledges the support he received from DAAD (Germany Academic Exchange Service), Addis Ababa University, and ISP(International Science Program) to conduct this research. The second author acknowledges Mathematics Department of Addis Ababa University for its financial support and allowing him to use the graduate Mathematics computer laboratory when he was conducting this work and also he would like to thank Bonga Colleges of Teachers Education for its support during his MSC study.

\section{References and Notes}

[1] A. Björner, Shellable and Cohen-Macaulay partially ordered sets, (1980), 159-183.

[2] R. Ehrenborg and M. Readdy, The Möbius function of partitions with restricted block sizes, 2007, 1-3

[3] Patricia Hersh, Lexicographic Shellability for Balanced Complexes, Department of Mathematics, University of Washington, Seattle, Revised March 4, 2002, 226-228

[4] Dmitry Kozlov, Combinatorial Algebraic Topology, 2007, 219-224

[5] Toufik Mansor, Combinatorics of Set Partitions, 2013, 1-5

[6] Brüggemann.R.Patil, Introduction To partial Order Application, 2011, 19-21

[7] S.A.Fufa, On the Möbius Function of Pointed Partitions and Exponential Pointed Structures, Doctoral dissertation, University of Addis Ababa, 2015, 37-39, 56-57

[8] S.A.Fufa; M.Zeleke, (2018): On Möbius Function of Pointed Graded Lattice. Indian J Pure Appl Math (2018) 49: 51.

[9] Jay Schweig and Russ Woodroofe, a Broad class of Shellable Lattices, 2016, 7-9 Press,Cambridge, 1999.

[10] R. P. Stanley, Enumerative Combinatorics I, Second Edition, Cambridge University Press, Cambridge, 2011, 277-280 